\title{
MobMaps: towards a shared environment for collaborative social activism
}

\author{
Luís Gens ${ }^{1}$, Hugo Paredes ${ }^{2}$, Paulo Martins ${ }^{2}$, Benjamim Fonseca ${ }^{3}$, Yishay Mor ${ }^{3}$, \\ Leonel Morgado ${ }^{2}$ \\ ${ }^{1}$ UTAD, Quinta de Prados, Apartado 1013, 5001-801 Vila Real, Portugal \\ luisgens@utad.pt \\ ${ }^{2}$ GECAD/UTAD, Quinta de Prados, Apartado 1013, 5001-801 Vila Real, Portugal \\ \{hparedes, pmartins, leonelm, benjaf\}@utad.pt \\ ${ }^{3}$ Institute of Education, University of London, London, UK \\ yishaym@gmail.com
}

\begin{abstract}
Nowadays it is possible to disseminate information to the all world in real time using current communication tools supported mostly by the Internet. The work of several organizations reporting a multitude of problems that our society faces can be sustained by participatory platforms, which stimulate the collaboration of participants all over the world. In this paper we present a technological platform that provides a shared environment for collaborative social activism. We adapted the platform to a particular organization, MachsomWatch that reports human rights abuses in Israelis checkpoints. Finally we present some preliminary results obtained by ethnographic research using the developed platform.
\end{abstract}

Keywords: mobile collaboration, georeferenced information, social activism

\section{Introduction}

In an era of accelerated evolution of knowledge, the difference between the success and failure of an initiative often depends on the amount and quality of information that one can get from the field and on the field. Technological progress enables the development of solutions to overcome some of the information constraints of field work. The proliferation of mobile devices such as cell phones and PDAs allows field operations to benefit from tools similar to those available for office-based work [10].

The Web as we know it is changing. New wiki and blogging sites are appearing constantly, and have contributed to further develop collaborative work. In our days, people like to participate, to share their opinions, their knowledge, and their culture using computer technologies. By introducing mobile devices into this mix we have mobile social software [1]. At the same time, we see a growing use of geographic information on the Web mostly due to low-cost availability of image and geographic data from map servers, either from public or private organizations. If we can find at our disposal these kinds of technology and people motivated to do collaborative social 
activism, why don't we take advantage of both of them? Why not put them working together and provide tools to overcome their problems?

On this paper we start by presenting some social activism scenarios that represent situations that range from everyday life to disaster events, as well as some issues that people face trying to deal with them. In this context we present our concept of social activism. A comprehensive analysis of existing technologies is given afterwards, followed by a technical presentation and an overview of a technological platform proposal. Afterwards an existing prototype implementation is presented associated with a case study. Finally, we conclude with some final remarks.

\section{Social activism scenarios}

In present days, our society faces a multitude of problems. These can vary from human rights violations, natural disasters such as floods, forest fires, earthquakes, etc., and even neglect for people's special needs (sight, hearing or motion limitations, for instance). Some of these are known worldwide, while others remain silent.

Reporting human rights abuses: Every year Amnesty International reports several situation of abuses of human rights around the world. A particular case of these situations occurs in Israel where every year several Non Governmental Organizations (NGO) report repeated human rights abuses performed by army and border police on Palestinians crossing checkpoints. Included in this kind of organizations is MachsomWatch, an Israeli women organization established in February 2001, with more than 500 members (all volunteers) of different backgrounds, personal and social characteristics. The organization monitors military checkpoints trying to resolve the situations and conflicts at the moment and in the local where they occur and after reporting the situations to the global community by making their reports available on the web. As a result of their efforts, the MachsomWatch members expect to improve the conditions in which people that have to use the checkpoints live. In spite of all their effort, there are some issues that can be very difficult to solve, like coordination between different agents on the field, the ability to record incidents, the need to provide rapid real-time response, and to let people all over the world know what is happening.

Urban barriers to accessibility: Many people suffer from limitations such as blindness, hearing loss, motion limitations, etc. For them, moving from place to place can be very challenging. Private and public buildings were built (and in many cases, still are) thinking only of people that do not have such limitations. If one compounds this with the typical problems of a city (street holes, cars parked on sidewalks, etc.), one ends up with an adverse environment. To help solve these problems, several organizations have sprung around the world, to act as a middle man between people and the entities responsible for solving this kind of problems.

Spatial Data Infrastructure applied to disaster management: Spatial data has won a special place in our day to day life. Today we find infrastructures based on spatial data that help on the decision-making level when applied in disaster management. These infrastructures are named Spatial Data Infrastructures (SDI). SDI has proven to be very important and essential in collaborative decision-making in 
disaster management [9]. Normally, these kinds of infrastructures are composed by different kinds of organisms and/or organizations, collecting and maintaining the database for responding to disasters collaboratively. The aim of the SID is to create an environment where users can retrieve, access, update and disseminate spatial data in an easy and secure way. By allowing the sharing of data, the SDI enables users to save time, efforts and resources.

The technological emergence of the Web into the Web 2.0 gave the people the power to construct their Web. This fact empowered their ability to report some of the above mentioned facts that occur all over the world, enjoying their free speech rights and their moral responsibilities as members of the society to reveal such situations.

The use of collaborative systems to solve situations that affect many people can have a very good impact on a community's ability to uphold rules and care for the wellbeing of community members. By supporting passive citizens to change into a more interactive role, collaborative systems promote a higher sense of community, making people more concerned about their surroundings [6]. Seeing how other people enter information to help someone else is a major incentive, and provides encouragement for others to do the same. Also, because people can now have a direct impact on the situation, they also have an increased concern to see it through, putting extra pressure on the authorities to solve problems more quickly.

\section{Technological background}

Some of the challenges of the previous scenarios can be addressed by a system that allows information to be taken to those who need it and collected from those who have it, allowing people to participate, sharing information and knowledge, taking advantage of collaborative work. If we allow people to collect, validate and correct information, we can have quick access to important information that can be used to improve one's actions under some situations [4].

With the massive proliferation of wikis on the Web and the easy access maps provided by free tools like Google Maps, the integration between the two technologies became natural. One of the best-known examples is Wikimapia, a Web site based on a wiki that provides a map interface, where anybody can search, add, and modify information on a certain location. Many other applications implement the same idea (using maps in connection with collaboration tools), e.g. OpenStreetMap and HealthMap. A particularly important example of how these tools can work together to achieve a very important and relevant goal is Scipionus (www.scipionus.com), which was used during the Katrina disaster for people to provide information about problems they were aware of in certain areas, and to allow others to collect news about their relatives, friends or colleagues[3]. This kind of systems, like any other based on large-scale collaboration efforts, is not without flaws, and always faces the same problem: validation of the information inserted in the platform [11].

The ability to use geospatial data retrieved from the field is very relevant to increase the quality of the information gathered [2]. Current tools have several 
limitations, specially regarding the ability to manipulate spatial data [10]. For greater use of this specific function, new mobile software tools are needed.

The use of mobile devices to allow access to spatial information (e.g., Google Maps Mobile) creates new services and situations for the users [12]. Some examples of integration between maps and mobile devices are PDPal, a spatial annotation application for the Palm PDA; and Yellow Arrow, an application to combine physical marks with information about them [1].

Mobile devices can be used, and are in fact being used, to retrieve information from the field and to add data to maps, allowing for a better understanding of the local and global situation. Tools like ProMed-mail, a tool to help find and distribute information about diseases regarding animals, plants or humans all over the world in a very quick manner [8], are changing the way many organizations respond to unexpected situations with their ability to gather and distribute data easily.

A new and important feature of cell phones is the digital camera. This device has considerably increased the range of uses of the cell phone as a collaborative tool, by providing a way to take pictures in the spur of the moment and to upload them in a single, continuous act. Specific software services, known as photo blogging or moblogging, allow users to use their cell phone cameras to take pictures and upload them to a site or blog where, later, commentaries can be added by any user. There's a wide variety of application in this segment, e.g. MobShare, Kodak Mobile, Buzznet, and Nokia Lifelog [5]. As another example, albeit without the ability to upload photos from the cell phone,is Flickr that (at this time) is the largest site for image sharing [5].

Mobile communication infrastructures are sufficiently diversified to allow at least a minimum level of access in almost every place populated by humans (e.g., satellite communication). They are the ideal platform to provide access to information and contribute with information. A platform based on three basic principles, mobility (providing generalized access and greater freedom of movements, since one does not need to be connected to a physical network to have access to the information), collaborative work, and location-based information, could be used to improve many social activism situations.

\section{MobMaps Platform}

Currently, there are very few tools that allow maps to be used on mobile devices and, at the same time, offering a way to do collaborative work. Our technological platform proposal tries to fill this gap. Our main objective was to develop a platform to serve as layer between mobile devices and geospatial data services. This platform needed to be device-independent: able to run in any kind of mobile device, be it a cell phone or a PDA.

Moreover, the MobMaps ${ }^{1}$ platform intends to be a generic platform for collaborative social activism that can be adapted to the several situations fulfilling specific requirements of each application context.

${ }^{1}$ MobMaps derives from the words "Mob" plus "Maps" empathizing the geo-referenciation collaborating people. 
The MobMaps technological platform has five main components: Mobile Devices; Application Layer; Input/Output Modules; Maps Server; and Information Server.

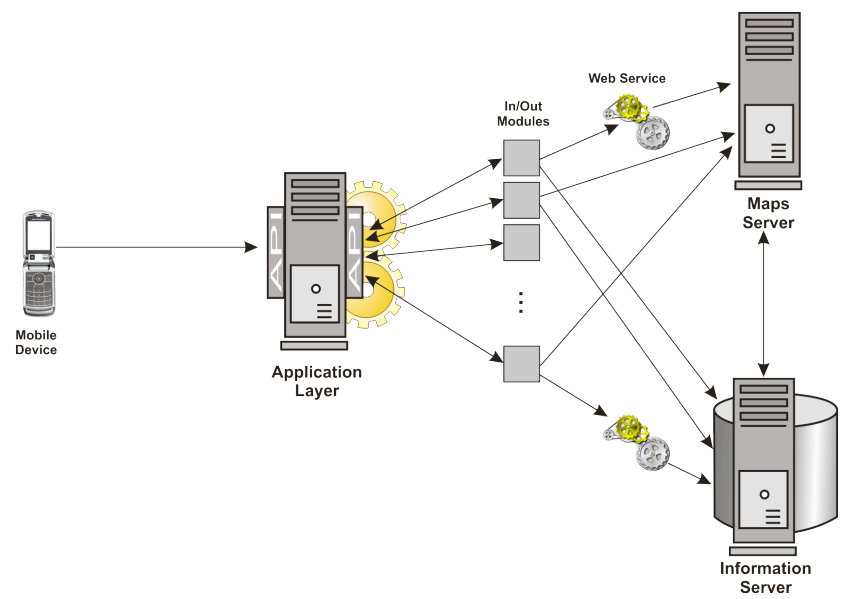

Figure 1 - MobMaps technological platform.

The Mobile Device application is composed of a module for presenting maps to the user, providing means for navigation, visualization of information and the necessary tools to allow data entry by the user.

The front-end mobile devices perform as little data processing as necessary for the user to access the application. The application can adapt itself to the features of each mobile device, in order to run on devices with different levels of hardware resources. Centralized content storage and access control are done at the Information Server and at the Maps Server (Figure 1). These can be developed specifically for use in a scenario, or readily available Web services, linked to the Application Layer through Input/Output modules.

The Application Layer can be called the heart of the entire system behaving as the functional core of the MobMaps platform. It is responsible for the coordination between all the different modules and for most of the needed information processing activities. Included in the major functions of this module are: translation coordination - convert values provided from the mobile device to accepted GIS coordinates; image resizing - adjust image sizes to the mobile devices' characteristics.

In order to provide some of these functionalities, the platform relies on a set of In/Out Modules, which allows the communication and data structure translation between the Appplication Layer and the backend layers composed of Information Server and Maps Server. These modules adapt the request provided from the Application Layer interface and translate that data into information that can be understood by the servers (the map and information server). Then, when the server returns the requested information, the reverse operation occurs. The selection by the Application Layer of the correct In/Out module to be used depends on the original request provided by the user. This middleware layer represents an important architectural implementation as it allows the system to work with all, or at least most, maps servers and information management systems available. 
The maps server that can be used with the platform can vary from the use of a complex GIS system to a simple implementation using of a still image. In the specification stages, our main concerns were the single images scenario and the GIS map server scenario. This choice was the result of a particular concern to work on solutions primarily based on largely accepted software or software related standards such as OGC, which guarantees the interoperability of the platform and a large amount of available maps servers systems.

Since MobMaps aimed to provide a way to record, search and access information in a collaborative environment, an Information System needs to record that information. The complexity of the Information System can vary from a simple text file to a very complicated and elaborated blog or wiki system. Despite the fact that nowadays the use of database based systems has reached almost every corner of the computer world, there are still some systems that, because of size limitation or due to their highly specific nature, have other information structures.

The Information System can have a full, partial or zero cooperation with the map server, depending on the nature of the specific service that is being used. Any and all of these different situations can be implemented with residual effort, thanks to our platform architecture.

\section{MachsomWatch: a case study}

MachsomWatch is, as stated before, an organization of Israeli women from different age, educational, social and economical ranges, that monitors military checkpoints and attempts to resolve and defuse the situations by reporting events on the Web. MachsomWatch volunteers need to be in movement as they act in groups of 2 or 3 volunteers in more than 30 checkpoints in shifts of 3 to 5 hours each day. When on action in the field, the volunteers do not have traditional Internet access to communicate with the head quarters of the organization and report the situations. In this context the most adequate and available communication tool are mobile phones.

Communication is needed in the resolution of certain situations, where local diplomacy with the observers and the militaries cannot solve the violations that occur. In that kind of situations, volunteers need to contact the organization headquarters in order to report and solve the problem. Despite the resolution of the problems, every situation that occurs in a checkpoint is reported and can be accessed in the organization's Web site (http://www.machsomwatch.org/en).

The adaptations of MobMaps platform to the specific case of MachsomWatch has as requirements: the need to be used by volunteers in the checkpoints, therefore a mobile application; must be user friendly, as volunteers are from different ages, social, economical and educational ranges; must enable the access at all time to registers about each checkpoint and the current status of all violations; and have the possibility to add new information in real time to the system as the situation occurs.

Based on these requirements the MobMaps platform was simplified in three main layers: Mobile Devices; Application Layer; and Information Server. As stated in the requirements, in this case study there are only a fixed area and a pre-defined number and locations of checkpoints where situations occurs, so the Maps Server is not 
needed. In this case a fixed map image is located in the Application Layer server and will be transformed according to the mobile characteristics that came in the requests from the mobile device. As far as the In/Out modules are concerned, the simplification of the platform and the adaptation to a specific case has as consequence the removal of architectural generalizations. In the case study the implementation of the mobile device makes 4 requests to the application layer (Figure 2): (1) map request; (2) checkpoint position request; (3) checkpoint information request; and (4) request to send new information about a checkpoint.
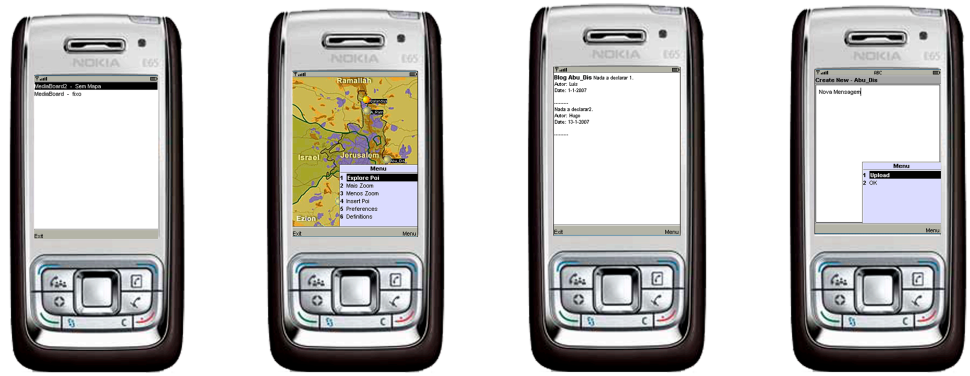

Figure 2 - Mobile application: (a) services menu, (b) map and options (c) checkpoint information and (d) upload screen.

The developed prototype was intended to be tested on the field, so a member of our research group traveled to Israel for 8 days where, using an ethnographic research methodology, he observed, reported and evaluated the working methods of the MachsomWatch. The trip was planned in order to fulfill three major objectives: present the platform to the organization; educate the future users of the platform; and follow some teams in their work at checkpoints.

The field study revealed some important conclusions about the working methodologies of the organization. The first is that the MachsomWatch neither have fixed headquarters nor a permanent team to assist the resolution of situations in the checkpoints. The second is the usage of mobile Internet in Israel, which is very expensive, so most of the volunteers do not have contracts with that functionality. The last conclusion was about the average age of the members of MachsomWatch: more than $85 \%$ of the members are more than 55 years old.

In what concerns research on the field four meetings with members of the organization were performed and the work of 5 volunteer teams on the field was observed, reported and analyzed, covering more than 11 chekpoints visits. The work was reported in the blog http://aventurasdeisrael.blogspot.com/ (in Portuguese).

\section{Conclusions and final remarks}

Large amounts of information are hard to process. Usually graphic methods of delivery are used to allow people to filter meaningful information. By seeing it on a map, people can understand how the global status evolved. Collaborative platforms provide a way for all people to share their knowledge creating possibilities for new, 
innovative approaches to the problem's resolution and can be seen as optimal informative platform. By using mobile devices, these people can access and deliver information in places that classic means of communication cannot reach.

In this paper we have presented some scenarios from real life situations, their problems and how a combination between spatial data, the use of mobile devices and collaborative work can help minimizing them. To allow this combination to work we have presented a technical proposal for porting the collaborative work to the field through the use of mobile devices, maximizing the response to the situations presented on those scenarios. This system addresses the problem of the lack of solutions that exists when people leave the office and need to work, either on the move or in open country. In the process, we have identified issues regarding the communications between mobile applications, map servers and information storing systems.

\section{References}

1. Bleecker, J. (2006, May). Design Patterns for Mobile Social Software. Paper presented at the meeting WWW2006 Workshop "MobEA IV - Empowering the Mobile Web", Edinburg, Scotland.

2. Christensen, U. (2001). Conventions and articulation work in a mobile workplace. SlGGROUP Bulletin December 2001 22(3), 16-21.

3. Church, R.L. (2002). Geographical information systems and location science. Computers \& Operations Research 29, 541-562.

4. Gens, L., Alves, H., Paredes, H., Martins, P., Fonseca, B., Bariso, E., Ramondt, L. Mor, Y. \& Morgado, L. (2007, September) Mobile software for gathering and managing georeferenced information from crisis areas. Paper presented at the meeting Proceedings of the Second International Conference on Internet Technologies and Applications (ITA 07), Newi, Wrexham, UK.

5. Jacucci, G., Oulasvirta, A., Salovaara, A. \& Sarvas (2005, November). Supporting the Shared Experience of Spectators through Mobile Group Media. Paper presented at the meeting Proceedings of Group 2005, Sanibel Island, Florida, USA.

6. Kalibo, H.W. and Medley, K.E. (2007). Participatory resource mapping for adaptive collaborative management at Mt. Kasigau, Kenya. Landscape and Urban Planning 82, 145158.

7. Kolbitsh, J. \& Maurer, H. (2006). The transformation of the Web: How Emerging Communities Shape the Information we Consume. Journal of Universal Computer Science 12(2), 187-213.

8. Madoff, L.C \& Woodall, J.P. (2005). The Internet and the Global Monitoring of Emerging Diseases: Lessons from the First 10 Years of ProMED-mail. Archives of Medical Research 36, 724-730.

9. Mansourian, A., Rajabifard, A., Zoej, M.J.V. \& Williamson, I. (2006). Using SDI and Webbased system to facilitate disaster management. Computers \& Geosciencies 32, 303-315.

10.Nusser, S. Miller, L., Clarke, K. \& Goodchild, M. (2003b). Geospatial IT for mobile field data collection. Communications of the ACM 46(1), 63-64.

11.Palen, L., Hiltz, S.R. \& Liu, S.B. (2007). Online forums supporting grassroots participation. Communications of the ACM 50(3), 54-58.

12.Tezuka, T., Kurashima, T. \& Tanaka, K. (2006, May). Toward Tighter Integration of Web Search with a Geographic Information System. Paper presented at the meeting WWW 2006, Edinburg, Scotland. 